$\mathrm{DE}$

M E D I C I N A

T R O P I C A L

$\mathrm{DE}$

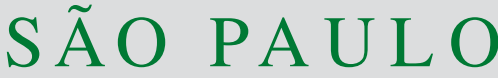

JOURNAL OF THE SÃO PAULO INSTITUTE OF TROPICAL MEDICINE

${ }^{1}$ Universidade Estadual de Campinas, Faculdade de Ciências Médicas, Laboratório de Pesquisa Aplicada em Dermatologia e Infecção por Bartonella, Campinas, São Paulo, Brazil

'Universidade Estadual de Campinas, Faculdade de Ciências Médicas, Departamento de Clínica Médica, Disciplina de Dermatologia, Campinas, São Paulo, Brazil

${ }^{3}$ Universidade Estadual de Campinas, Faculdade de Ciências Médicas, Departamento de Clínica Médica, Disciplina de Moléstias Infecciosas, Campinas, São Paulo, Brazil

${ }^{4}$ Universidade Estadual de Campinas, Faculdade de Ciências Médicas, Departamento de Patologia, Campinas, São Paulo, Brazil

Correspondence to: Paulo Eduardo Neves Ferreira Velho

Universidade Estadual de Campinas, Faculdade de Ciências Médicas, Departamento de Clínica Médica, Disciplina de Dermatologia, Rua Tessália Vieira de Camargo, 126, Cidade Universitária Zeferino Vaz, CEP 13083-887, Campinas, SP, Brazil

Tel: +55 1935219134

E- mail: pvelho@unicamp.br

Received: 1 October 2021

Accepted: 26 January 2022

\section{Chronic type 2 reaction possibly triggered by an asymptomatic Bartonella henselae infection in a leprosy patient}

\author{
Luciene Silva dos Santos ${ }^{\circledR}$, Marina Rovani Drummond ${ }^{\circledR}$, Andrea \\ Fernandes Eloy da Costa França ${ }^{2}$, Maria Helena Postal Pavan ${ }^{3}$, Rafael \\ Fantelli Stelini ${ }^{4}$, Maria Letícia Cintra ${ }^{1}{ }^{4}$, Elemir Macedo de Souza ${ }^{2}$, Paulo \\ Eduardo Neves Ferreira Velho ${ }^{1,2}$
}

\section{ABSTRACT}

As leprosy and leprosy reactions are the most prevalent infectious cause of physical disability, it is important to commit efforts to better understand these chronic reactions. Infections, even when asymptomatic, can trigger leprosy reactions and Bartonella spp. in turn, can cause chronic infections. We presented a case of a 51-year-old man who was admitted presenting with chronic type 2 leprosy reactions. He had a lepromatous form of leprosy that was histologically diagnosed six months after the onset of signs and symptoms compatible with a chronic type 2 reaction. He reported a history of a previous hepatitis B diagnosis. During a 24-month multidrug therapy (MDT), chronic reactions were partially controlled with prednisone and thalidomide. Thirty-three months following the leprosy treatment, he still experienced chronic reactions, and whole bacilli as well as globi were found on a new skin biopsy. Since coinfections can trigger type 2 reactions and the patient had close contact with animals and ticks, we investigated the presence of a Bartonella sp. infection. Bartonella henselae DNA was detected in a skin fragment obtained before the beginning of the leprosy retreatment. However, even after six months of a second leprosy MDT, he continued to experience type 2 chronic reactions. He was admitted to the hospital to undergo an intravenous antibiotic therapy for 14 days and then complete the treatment per os for ten more weeks. Leprosy reactions improved following the treatment for B. henselae. After completing the MDT treatment, he has been accompanied for sixty months with no signs of leprosy or leprosy reactions. The asymptomatic infection by $B$. henselae in this patient was considered the putative trigger of chronic leprosy reactions and leprosy relapse.

KEYWORDS: Bartonella. Coinfection. Leprosy. Erythema nodosum.

\section{CASE REPORT}

A 51-year-old Caucasian rodeo cowboy was admitted to the University of Campinas Hospital with chronic type 2 leprosy reactions. He had been diagnosed with leprosy two years earlier after a six-month period of nodular skin lesions, lymph node enlargement and night sweats. Although he had no history of contact with leprosy, this diagnosis was clinically and histologically confirmed. He was treated with a multibacillary (MB) regimen recommended by the WHO multidrug therapy (MDT), but dapsone was replaced in the first month of treatment by ofloxacin $400 \mathrm{mg}$ daily due to anemia, following the Brazilian Department of Health recommendations ${ }^{1}$. The type 2 reaction (T2R) was treated with prednisone 
(maximum of $100 \mathrm{mg}$ daily) and thalidomide (maximum of $200 \mathrm{mg}$ daily) for the two years of treatment of leprosy. He had also been diagnosed with hepatitis B (HB) and was treated with tenofovir but maintained the leprosy reactions during all this time.

He presented with erythematous-nodular lesions (ENL), some with central scars on the buttocks and the thighs. He also had madarosis and ear lobe induration. The cubital and popliteal nerves were thickened and there was tactile hypoesthesia in boots and gloves. His liver and spleen were palpable at 3 and $2 \mathrm{~cm}$ from the coastal borders. He was receiving $100 \mathrm{mg}$ of thalidomide and $300 \mathrm{mg}$ of tenofovir, besides vitamin $\mathrm{D}$, calcium carbonate and omeprazole. The patient used rifampicin doses once a month, and the other medications daily.

Odontological evaluation, a tuberculin test and serological tests for paracoccidioidomycosis, brucellosis, syphilis, toxoplasmosis, mononucleosis, AIDS and hepatitis $\mathrm{C}$ were requested because of the chronic leprosy reactions, and fever episodes related to ENL. All the investigations were negative or within normal limits. The periodontal disease was treated. Relatives were evaluated and none had leprosy.

A liver biopsy showed cirrhosis with formation of regenerative pseudo nodules with moderate septal lymphocytic infiltrate. An upper gastrointestinal endoscopy revealed thin gauge esophageal varices, thus classifying him as Child A. The viral load for HB virus was undetectable in his peripheral blood sample. The treatment for HB was replaced by $0.5 \mathrm{mg}$ of entecavir daily because the patient presented hematuria with erythrocyte dysmorphism that improved after treatment change.

During thirty-three months after the end of MDT, the patient presented with several episodes of ENL that were treated with thalidomide (up to $300 \mathrm{mg}$ daily), and neuritis, that improved with high doses of prednisone (up to $60 \mathrm{mg}$ daily). After this period, he presented with bullous lesions without nodulation on the lower limbs suggestive of erythema multiform leprosy reaction. The histopathological examination revealed a marked upper dermis edema, blisters and ulcers. An intense dermo-hypodermal foamy cell infiltrate contained a number of entire fast acid bacilli (FAB), some globi (clumps of bacilli) and copious comminuted bacilli (Figure 1), confirming that the patient had ENL. The Mycobacterium leprae gene resistance analysis was not available at the hospital, at that time.

Even though the patient had no bacillary angiomatosis lesion, as the patient was a rodeo cowboy and reported frequent contact with animals and ticks, Bartonella spp. was considered a possible cause of asymptomatic infection that could trigger and maintain the chronic T2R in a patient with leprosy. A new skin biopsy was performed and sent to microbiological and molecular testing. Following the described histopathological examination, MDT was restarted with rifampicin, clofazimine and ofloxacin, following the Brazilian Department of Health's recommendation (dapsone could not be used due to the anemia in the patient) ${ }^{1}$. DNA was extracted from the skin fragment and its liquid culture. Bartonella sp. DNA was recovered from the culture sample by conventional PCR targeting the ITS region and the $s s r A$ gene $^{2}$. Both amplicons revealed $100 \%$ homology with Bartonella henselae (access to GenBank BX897699.1). Thereafter, two blood samples were collected with 13 days of interval and B. henselae DNA was also detected in both. Doxycycline $200 \mathrm{mg} / \mathrm{d}$ was prescribed orally, but the patient discontinued it because of diarrhea.

Six months after the beginning of the second MDT, the patient was receiving thalidomide $100 \mathrm{mg} / \mathrm{d}$ and was admitted to receive intravenous antibiotic therapy composed of $80 \mathrm{mg}$ of gentamicin and $1 \mathrm{~g}$ of amoxicillin in addition to $200 \mathrm{mg}$ clavulanate three times a day for 14 days. At discharge, he was receiving thalidomide $100 \mathrm{mg} / \mathrm{d}$ and $500 \mathrm{mg}$ of azithromycin were prescribed once a day for up to six weeks. From the first follow-up visit 15 days after hospital discharge and after, he reported no more evidence of ENL. Thalidomide was reduced to $100 \mathrm{mg}$ every other day and subsequently discontinued. We prescribed $200 \mathrm{mg}$

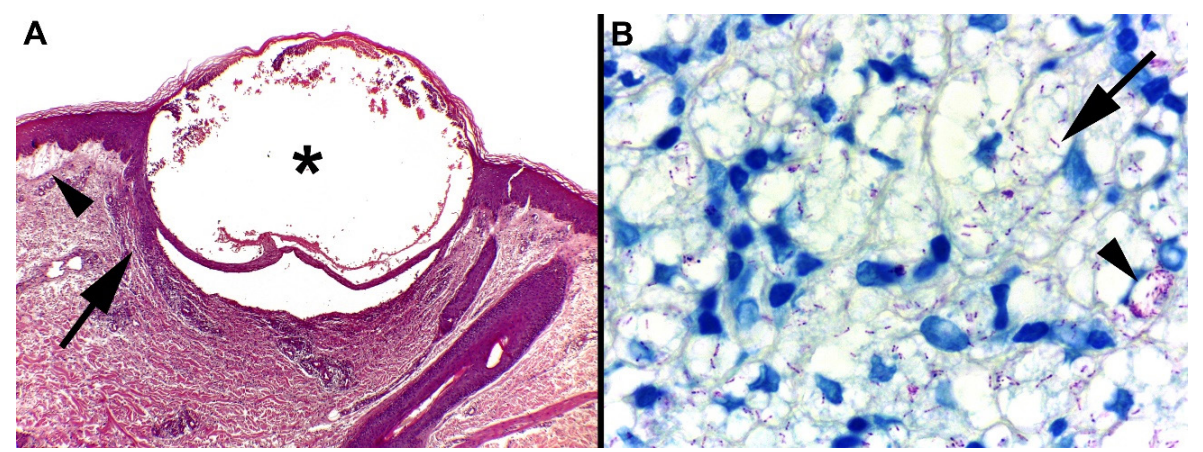

Figure 1 - A) subepidermal pustule (asterisk) undergoing re-epithelization (arrow) and a marked upper dermis edema (arrowhead); B) an intense hypodermal foamy cell infiltrate containing a number of entire fast acid bacilli (arrow) and some globi (arrowhead). A) $H \& E$, original magnification $\times 40$; B) Fite Faraco, original magnification $\times 1,000$. 


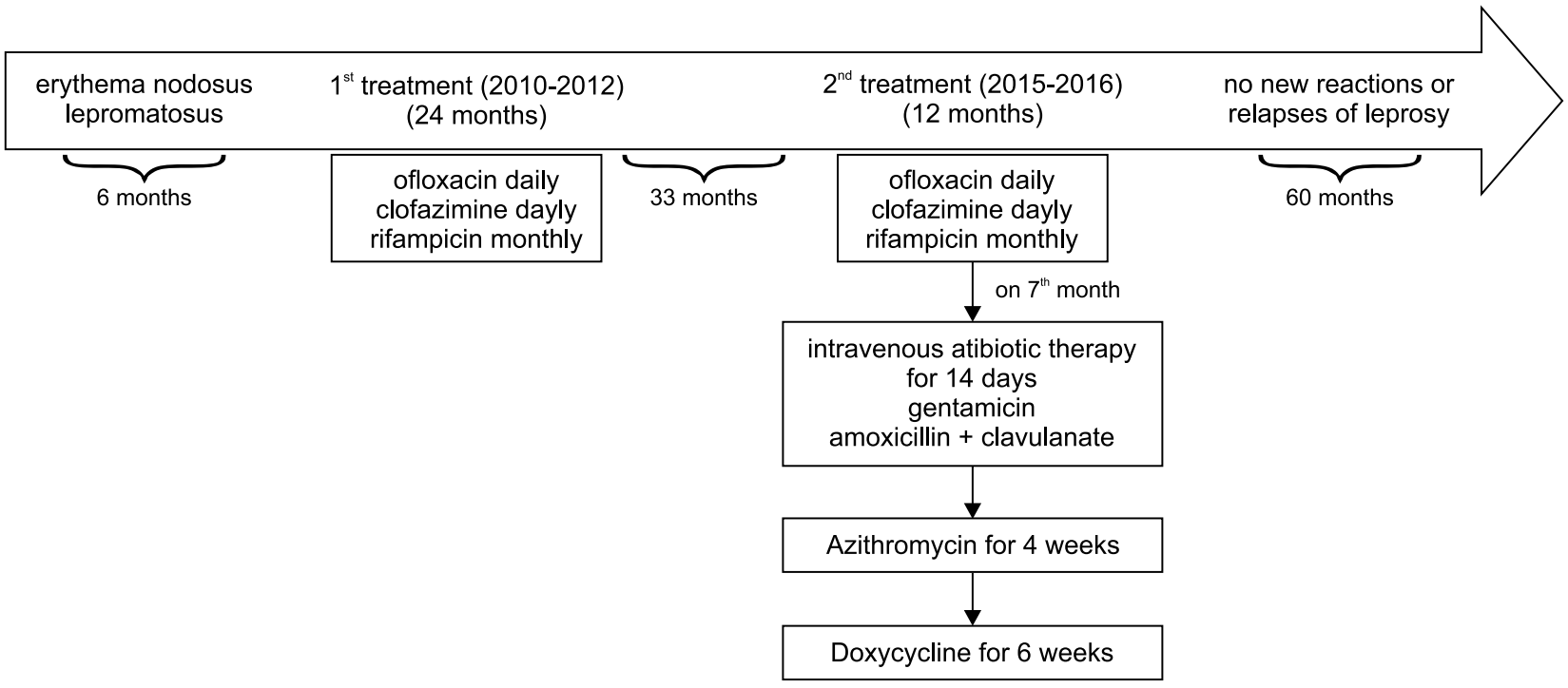

Figure 2 - Timeline treatment of a 51-year-old patient with chronic type 2 leprosy reactions possibly triggered by an asymptomatic $B$. henselae infection.

daily of doxycycline for another six weeks and the patient tolerated the antibiotic (Figure 2). A 12-month MDT was completed. Sixty months after the second MDT, the patient presented with no leprosy reactions or signs of relapses. At the moment the patient is only on antiviral medication for hepatitis.

\section{DISCUSSION}

Leprosy is an ancient disease related to poverty and is also the main cause of infectious physical disability. The disease is still a public health problem, especially in low- and middle-income countries such as India, Brazil and Indonesia ${ }^{3}$. Leprosy is often diagnosed late as many physicians have little experience with its clinical presentation. In Brazil, physical disabilities are most frequent in regions with lower prevalence of the disease, suggesting a later diagnosis by physicians in the regions where the disease is less common (Figure 3$)^{4}$.

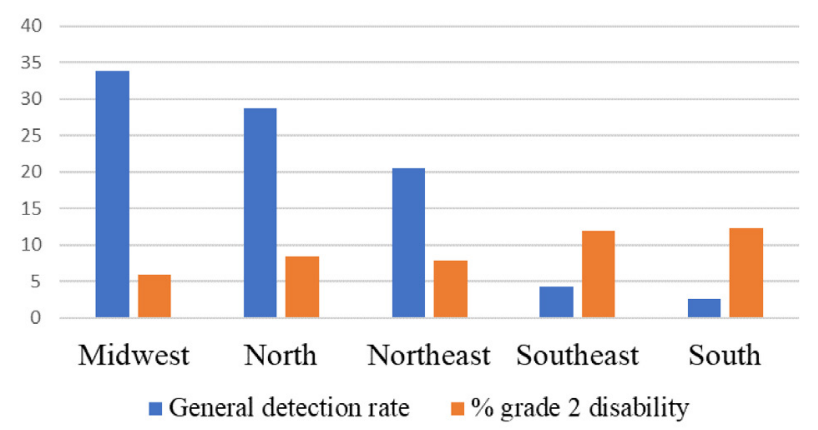

Figure 3 - Epidemiological indicators of leprosy (general detection rate and percentage of grade 2 disability) according to the Brazilian region, $2017^{4}$.
Acute inflammatory phenomena are called leprosy reactions which also dependent on the genetically inherited immune capacity. Leprosy reactions do not always depend on the active infection by M. leprae. Reactional outbreaks are serious, as there is an exacerbation of the inflammatory condition. These cause irreversible and more serious damage than the disease itself and could be reversed if treated on time. They are considered clinical urgencies because of the risk of physical disability ${ }^{5}$. It is estimated that on average 30 to $35 \%$ of patients develop leprosy reactions, they are more common and frequent in multibacillary patients, reaching the rate of $50 \%$. Patients who experience reactions during treatment are more likely to develop reactions after the end of the specific treatment ${ }^{5,6}$.

Type 1 (cellular) and type 2 (humoral) leprosy reactions are usually self-limited manifestations ${ }^{6}$. Several factors can trigger these reactions, especially for type 2 leprosy. Identifying and tracking these factors is important for the prevention and control of these episodes. In general, pregnancy, childbirth, stress, alcoholism or concomitant infections trigger these reactions. The infections are major precipitants ${ }^{6}$ as they can stimulate the production of immunological mediators that can trigger, aggravate or maintain reaction outbreaks ${ }^{6}$. Patients with viruses such as human immunodeficiency virus (HIV), human T cell lymphotrophic virus type 1 (HTLV-1), hepatitis B virus (HBV) and hepatitis $\mathrm{C}$ virus (HCV) have higher rates of neuritis and nerve function impairment than non-coinfected leprosy subjects. Urinary tract infection and periodontitis also stand out as reaction triggers 5 . This patient presented with periodontal disease and viral hepatitis B. Coinfection in leprosy is very a very important issue to be investigated as 
it often occurs in neglected diseases with late misdiagnosis, leading to physical disabilities and even death.

Although a type 2 leprosy reaction may occur in a single episode, $33 \%$ to $64 \%$ of patients with this type of reaction may manifest it more than once. Among these patients, $31 \%$ may manifest five or more episodes over a period of more than two years, consequently increasing the risk of acquiring some physical disability ${ }^{7}$. Therefore, the duration of the reaction, the number of recurrences and the interval between them are important factors to consider.

Chronic reactions (new episodes beginning before the previous has ended) are rare. Kumar et al. ${ }^{7}$ define chronic reactions as persistent T2R for more than six months. In this case, neural involvement is common, requiring treatment with corticosteroids, associated or not with thalidomide, often in high doses ${ }^{5,7}$.

Even asymptomatic chronic coinfections are related to chronic leprosy reactions and leprosy relapses ${ }^{5}$. Bartonella spp. are neglected bacteria that usually cause chronic infections on their hosts. In humans, the infection can be asymptomatic, as has been documented in blood donors ${ }^{8}$. Bartonella henselae is the species most related to clinical manifestations in humans ${ }^{8}$.

We report a case of chronic leprosy reactions that were difficult to control. The patient had been treated for lepromatous leprosy with a multibacillary scheme for 24 months. He was coinfected with the HB virus and maintained T2Rs during and following the treatment. For 33 months, with his HB virus infection under control, he maintained the dependence on thalidomide and prednisone for chronic reactions. A new histological examination revealed whole bacilli and the presence of globi. Even with a second six-month multibacillary treatment, the chronic reactions remained until the beginning of an intravenous therapy for the newly diagnosed $B$. henselae infection. This species occurs worldwide and is the most disease-related species in humans. Cats and dogs are the most important reservoirs of this species, but the infection has already been associated with cows. Ectoparasites such as flies and ticks are vectors for $B$. henselae transmission ${ }^{9}$.

Although the infection may be asymptomatic in immunocompetent patients, $B$. henselae can cause granulomatous lymphadenopathy with or without fever. In these individuals, the condition is usually self-limiting, without the need of drugs for its improvement. It commonly occurs in cat scratch disease (CSD).

In immunodeficient patients, whether due to health problems or continuous use of immunosuppressants, Bartonella infection can be more severe or even fatal, as in these cases, the disease is more likely to spread systemically. AIDS patients, when infected by Bartonella, have higher bacterial load levels in circulation which develop more frequently than in immunocompetents, bacillary angiomatosis, hepatic or splenic peliosis, among other manifestations ${ }^{10-12}$. Similarly, several additional complications are reported in patients submitted to solid organ transplantation, especially kidney and liver ${ }^{13}$. It is also important to point out the increase of these infections in patients under immunosuppression to treat diseases like lupus and rheumatoid arthritis ${ }^{14,15}$. In the case of our patient, the chronic use of corticosteroids may have increased the pre-existing $B$. henselae bacteremia.

Coinfection of hepatitis B virus and $B$. henselae had been previously reported ${ }^{16}$, and this Bartonella species has already been associated with other liver manifestations, such as angiomatosis, peliosis, granulomatous reaction and even nonspecific hepatitis ${ }^{17}$.

Asymptomatic Bartonella sp. infection is described in blood donors, in inter febrile phases of trench fever, caused by Bartonella quintana, after the febrile phase and cutaneous manifestation of Carrion's disease, caused by Bartonella bacilliformis ${ }^{9}$.

There are no prospective studies on therapeutic regimens for the treatment of systemic bartonelloses. Oral azithromycin for five days is recommended for patients with $\mathrm{CSD}^{18}$. The suggested treatment for endocarditis by Bartonella sp. is intravenous gentamicin for two weeks, and six weeks of oral doxycycline ${ }^{19}$. Spach ${ }^{20}$ suggests doxycycline treatment for three months if the patient did not undergo valve replacement. If treatment with doxycycline is not possible, combination therapy with azithromycin should be followed by a total of six months of azithromycin. The patient we report here has improved from the chronic leprosy reactions with the administration of intravenous gentamicin and amoxicillin in addition to clavulanate for two weeks, azithromycin for more four weeks and doxycycline for another six weeks. The patient received, during only 12 months, the same MDT scheme he had used before for 24 months with no more reactions or relapses during a 60-month follow-up period.

This report describes, for the first time, the coinfection of $M$. leprae and $B$. henselae and a possible association between Bartonella sp. infection and chronic reactions or relapses in patients who have or had leprosy.

\section{AUTHORS' CONTRIBUTIONS}

LSS and MRD: literature search, study design, data collection, data analysis, data interpretation, writing; AFECF: data collection, data analysis, data interpretation, writing; MHPV, RFS, MLC and EMS: data analysis, data interpretation, writing; PENFV: literature search, study 
design, data collection, data analysis, data interpretation, writing.

\section{CONFLICT OF INTERESTS}

All authors declare that there is no conflict of interests.

\section{FUNDING}

A PhD scholarship by the Brazilian National Council for Scientific and Technological Development (CNPq) process $N^{\circ} 170501 / 2018-3$ (LSS); Postdoctoral fellowship by Sao Paulo Research Foundation (FAPESP) process N $^{\circ}$ 2018/12565-6 (MRD); Productivity Grants by the Brazilian National Council for Scientific and Technological Development (CNPq) process No 306970/2018-0 (PENFV).

\section{REFERENCES}

1. Brasil. Ministério da Saúde. Secretaria de Vigilância em Saúde. Departamento de Vigilância das Doenças Transmissíveis. Diretrizes para vigilância, atenção e eliminação da hanseníase como problema de saúde pública: manual técnico-operacional. Brasília: Ministério da Saúde; 2016.

2. Drummond MR, Visentainer L, Almeida AR, Angerami RN, Aoki $\mathrm{FH}$, Velho PE. Bartonella henselae bacteremia diagnosed postmortem in a myelodysplastic syndrome patient. Rev Inst Med Trop Sao Paulo. 2019;61:e50.

3. World Health Organization. Weekly Epidemiological Record, 2019, vol. 94, 35/36. Geneva: WHO; 2019.

4. Brasil. Ministério da Saúde. Secretaria de Vigilância em Saúde. Hanseníase 2022. Bol Epidemiol. 2022;N Esp:1-51. [cited 2022 Jan 28]. Available from: https://www.gov.br/saude/ pt-br/centrais-de-conteudo/publicacoes/boletins/boletinsepidemiologicos/especiais/2022/boletim-epidemiologico-dehanseniase- -25-01-2022.pdf

5. Machado PR, Machado L, Shibuya M, Rego J, Johnson W, Glesby MJ. Viral co-infection and leprosy outcomes: a cohort study. PLoS Negl Trop Dis. 2015;9:e0003865.

6. Polycarpou A, Walker SL, Lockwood DN. A systematic review of immunological studies of erythema nodosum leprosum. Front Immunol. 2017;8:233.

7. Kumar B, Dogra S, Kaur I. Epidemiological characteristics of leprosy reactions: 15 years experience from North India. Int J Lepr Other Mycobact Dis. 2004;72:125-33.
8. Pitassi LH, Diniz PP, Scorpio DG, Drummond MR, Lania $\mathrm{BG}$, Barjas-Castro ML, et al. Bartonella spp. bacteremia in blood donors from Campinas, Brazil. PLoS Negl Trop Dis. 2015;9:e0003467.

9. Tsai YL, Chang CC, Chuang ST, Chomel BB. Bartonella species and their ectoparasites: selective host adaptation or strain selection between the vector and the mammalian host? Comp Immunol Microbiol Infect Dis. 2011;34:299-314.

10. Diniz LM, Medeiros KB, Landeiro LG, Lucas EA. Bacillary angiomatosis with bone invasion. An Bras Dermatol. 2016;91:811-4.

11. Mosepele M, Mazo D, Cohn J. Bartonella infection in immunocompromised hosts: immunology of vascular infection and vasoproliferation. Clin Dev Immunol. 2012;2012:612809.

12. Magwai MG, Andronikou S. Isolated splenic peliosis in an immunocompromised patient. S Afr J Surg. 2012;50:92.

13. Ishikawa T, Suzuki T, Shinoda M, Takashi H, Yamaguchi H, Suzuki T, et al. A case of hepatosplenic cat scratch disease. Nihon Shokakibyo Gakkai Zasshi. 2006;103:1050-4.

14. Vargas-Hitos JA, Sabio JM, Navarrete-Navarrete N, Arenas-Miras MM, Zamora-Pasadas M, Jiménez-Alonso J. Cat scratch disease in an immunosuppressed patient with systemic lupus erythematosus. Lupus. 2016;25:310-1.

15. Orden AO, Nardi NN, Vilaseca AB, Colombini AC, Barrios NG, Vijnovich Baron A. Cat scratch disease during etanercept therapy in a rheumatoid arthritis patient. Reumatol Clin. 2018;14:303-6.

16. Kaçar N, Tasli L, Demirkan N, Ergin C, Ergin S. HIV-negative case of bacillary angiomatosis with chronic hepatitis B. J Dermatol. 2010;37:722-5.

17. Liston TE, Koehler JE. Granulomatous hepatitis and necrotizing splenitis due to Bartonella henselae in a patient with cancer: case report and review of hepatosplenic manifestations of bartonella infection. Clin Infect Dis. 1996;22:951-7.

18. Bass JW, Freitas BC, Freitas AD, Sisler CL, Chan DS, Vincent JM, et al. Prospective randomized double blind placebo-controlled evaluation of azithromycin for treatment of cat-scratch disease. Pediatr Infect Dis J. 1998;17:447-52.

19. Baddour LM, Wilson WR, Bayer AS, Fowler VG Jr, Tleyjeh IM, Rybak MJ, et al. Infective endocarditis in adults: diagnosis, antimicrobial therapy, and management of complications: a scientific statement for healthcare professionals from the American Heart Association. Circulation. 2015;132:1435-86.

20. Spach DH. Endocarditis caused by Bartonella. [cited 2022 Jan 28]. Available from: https://www.uptodate.com/contents/ endocarditis-caused-by-bartonella 